\title{
Invariant Solutions of a Nonlinear System of Differential Equations for Electromagnetic Field
}

Lyudmyla L. BARANNYK

Institute of Mathematics of the National Academy of Sciences of Ukraine, 3, Tereshchenkivska Street, 252004, Kyïv, Ukraine

\begin{abstract}
Solutions invariant under subalgebras of the affine algebra $A I G L(3, \mathbb{R})$ are found.
\end{abstract}

\section{Introduction}

The system of nonlinear differential equations

$$
\frac{\partial E_{k}}{\partial t}+H_{l} \frac{\partial E_{k}}{\partial x_{l}}=0, \quad \frac{\partial H_{k}}{\partial t}+E_{l} \frac{\partial H_{k}}{\partial x_{l}}=0 \quad(k, l=1,2,3)
$$

was proposed in [1] to study electromagnetic fields. For $E_{k}=H_{k}(k=1,2,3)$ system (1) becomes the Euler system of equations for ideal fluid that was studied in [2-6]. Symmetry properties of system (1) were investigated in [7]. It was stated that the maximal invariance algebra of the system under consideration is the affine algebra $\operatorname{AIGL}(4, \mathbb{R})$, three basis elements of which are nonlinear differential operators.

The aim of our research is to construct solutions of system (1) with the help of symmetry reduction of this system to systems of ordinary differential equations. In this paper, we restrict ourselves to the case where $E_{k}, H_{k}$ don't depend on the variable $x_{3}$.

Let us give a more detailed characteristic of each Section of the paper.

In Section 2, we get a number of statements concerning systems of linear invariants of subalgebras of the algebra $A I G L(4, \mathbb{R})$, on which we construct ansatzes $[8,9]$. Note that overwhelming majority of classes of conjugate subalgebras contains subalgebras for which there exist linear ansatzes.

In Section 3, we obtain solutions to system (1) that are functions only of $t$ and $x_{1}$. In this case, we can consider functions $E_{1}, H_{1}$ as components of a solution to the system of equations

$$
\frac{\partial E_{1}}{\partial t}+H_{1} \frac{\partial E_{1}}{\partial x_{1}}=0, \quad \frac{\partial H_{1}}{\partial t}+E_{1} \frac{\partial H_{1}}{\partial x_{1}}=0,
$$

functions $E_{2}, E_{3}$ as solutions of the homogeneous equation

$$
\frac{\partial E_{k}}{\partial t}+H_{1} \frac{\partial E_{k}}{\partial x_{1}}=0
$$


and functions $H_{2}, H_{3}$ as solutions of the homogeneous equation

$$
\frac{\partial H_{k}}{\partial t}+E_{1} \frac{\partial H_{k}}{\partial x_{1}}=0
$$

System of equations (2) is invariant under the affine algebra $A I G L(2, \mathbb{R})$. For this reason, with the help of one-dimensional subalgebras of the algebra $A I G L(2, \mathbb{R})$, one can reduce system (2) to systems of ODEs. If the function $E_{1}\left(t, x_{1}\right)$ is not constant, then each solution of equation (3) can be presented in the form $\psi\left(E_{1}\left(t, x_{1}\right)\right)$, where $\psi$ is some differentiable function. Moreover, for each differentiable function $\psi$, the function $\psi\left(E_{1}\left(t, x_{1}\right)\right)$ is a solution of equation (3). One can say the same about equation (4).

In Section 4, we construct solutions to system (1), that are functions of $t, x_{1}, x_{2}$. Functions $E_{1}, E_{2}, H_{1}, H_{2}$ are components of a solution to the system of equations

$$
\frac{\partial E_{k}}{\partial t}+H_{l} \frac{\partial E_{k}}{\partial x_{l}}=0, \quad \frac{\partial H_{k}}{\partial t}+E_{l} \frac{\partial H_{k}}{\partial x_{l}}=0 \quad(k, l=1,2),
$$

function $E_{3}$ can be regarded as a solution of the homogeneous equation

$$
\frac{\partial E_{3}}{\partial t}+H_{1} \frac{\partial E_{3}}{\partial x_{1}}+H_{2} \frac{\partial E_{3}}{\partial x_{2}}=0
$$

and $H_{3}$ as a solution of the homogeneous equation

$$
\frac{\partial H_{3}}{\partial t}+E_{1} \frac{\partial H_{3}}{\partial x_{1}}+E_{2} \frac{\partial H_{3}}{\partial x_{2}}=0 .
$$

If the functions $E_{1}\left(t, x_{1}, x_{2}\right), E_{2}\left(t, x_{1}, x_{2}\right)$ are functionally independent in some domain $\Gamma$, then each solution to equation (6) can be presented in this domain in the form $\psi\left(E_{1}\left(t, x_{1}, x_{2}\right), E_{2}\left(t, x_{1}, x_{2}\right)\right)$, where $\psi$ is some differentiable function. In addition, for an arbitrary differentiable function, $\psi$ the function $\psi\left(E_{1}\left(t, x_{1}, x_{2}\right), E_{2}\left(t, x_{1}, x_{2}\right)\right)$ of the variables $t, x_{1}, x_{2}$ is a solution of equation (6).

System of equations (5) is invariant under the affine algebra $A I G L(3, \mathbb{R})$. To perform reduction of system (5) to systems of ODEs, we need two-dimensional subalgebras of the algebra $\operatorname{AIGL}(3, \mathbb{R})$, that have only one main invariant of the variables $t, x_{1}, x_{2}$. We obtain the list of such subalgebras up to affine conjugation from results of the classification performed in [10].

\section{Linear ansatzes}

To unify systems (1), (2) and (5), we consider the system of equations

$$
\frac{\partial E_{k}}{\partial x_{0}}+H_{l} \frac{\partial E_{k}}{\partial x_{l}}=0, \quad \frac{\partial H_{k}}{\partial x_{0}}+E_{l} \frac{\partial H_{k}}{\partial x_{l}}=0 \quad(k, l=1,2, \ldots, n) .
$$

Here $x_{0}=t, n$ is an arbitrary natural number. By the same reasoning as in [7], we deduce that the invariance algebra of system $(7)$ is the affine algebra $A I G L(n+1, \mathbb{R})$, the basis of which is generated by the vector fields:

$$
\begin{aligned}
& P_{\mu}=\frac{\partial}{\partial x_{\mu}} \quad(\mu=0,1, \ldots, n), \quad \Gamma_{a b}=x_{a} \frac{\partial}{\partial x_{b}}+E_{a} \frac{\partial}{\partial E_{b}}+H_{a} \frac{\partial}{\partial H_{b}}, \\
& G_{a}=x_{0} \frac{\partial}{\partial x_{a}}+\frac{\partial}{\partial E_{a}}+\frac{\partial}{\partial H_{a}}, \quad G_{a}^{\prime}=x_{a} \frac{\partial}{\partial x_{0}}-E_{a} E_{l} \frac{\partial}{\partial E_{l}}-H_{a} H_{l} \frac{\partial}{\partial H_{l}}, \\
& \Lambda=x_{0} \frac{\partial}{\partial x_{0}}+x_{l} \frac{\partial}{\partial x_{l}} \quad(a, b=1,2, \ldots, n) .
\end{aligned}
$$


Mark that summation over the repeated index $l$ is from 1 to $n$.

The linear span of the system of operators, obtained from basis (8) as a result of excluding operators $G_{a}^{\prime}(a=1,2, \ldots, n)$, forms a Lie subalgebra of the algebra $A I G L(n+$ $1, \mathbb{R})$. Denote this subalgebra by $Q$ and call it the linear part of the invariance algebra of system (7).

Each operator $Y \in Q$ can be presented in the form

$$
Y=a_{\alpha}(x) \frac{\partial}{\partial x_{\alpha}}+b_{i j}\left(E_{j} \frac{\partial}{\partial E_{i}}+H_{j} \frac{\partial}{\partial H_{i}}\right)+c_{i}\left(\frac{\partial}{\partial E_{i}}+\frac{\partial}{\partial H_{i}}\right),
$$

where $x=\left(x_{0}, x_{1}, \ldots, x_{n}\right) ; b_{i j}, c_{i}$ are real numbers; $\alpha=0,1,2, \ldots, n ; i, j=1,2, \ldots, n$.

Definition. An invariant of the subalgebra $Q$ that is a linear function of variables $E_{a}$, $H_{a}(a=1, \ldots, n)$ is called linear.

Let

$$
\begin{aligned}
B & =\left(\begin{array}{cccc}
b_{11} & b_{12} & \ldots & b_{1 n} \\
b_{21} & b_{22} & \ldots & b_{2 n} \\
\ldots \ldots \ldots \ldots \ldots \ldots & \ldots \\
\ldots & \ldots & \ldots & \ldots \\
b_{n 1} & b_{n 2} & \ldots & b_{n n}
\end{array}\right), \quad C=\left(\begin{array}{c}
c_{1} \\
c_{2} \\
\vdots \\
c_{n}
\end{array}\right), \\
U & =\left(\begin{array}{cccc}
u_{11}(x) & u_{12}(x) & \ldots & u_{1 n}(x) \\
u_{21}(x) & u_{22}(x) & \ldots & u_{2 n}(x) \\
\ldots \ldots \ldots \ldots \ldots \ldots & \ldots \ldots \ldots \ldots \ldots \\
u_{n 1}(x) & u_{n 2}(x) & \ldots & u_{n n}(x)
\end{array}\right), \quad V=\left(\begin{array}{c}
v_{1}(x) \\
v_{2}(x) \\
\vdots \\
v_{n}(x)
\end{array}\right) .
\end{aligned}
$$

Theorem. A system of functions $f_{q}=u_{q i}(x) E_{i}+v_{q}(x), q=1,2, \ldots, n$, is a system of linear invariants of an operator $Y$, functionally independent of variables $E_{1}, E_{2}, \ldots, E_{n}$ if and only if

$$
a_{\alpha}(x) \frac{\partial U}{\partial x_{\alpha}}+U B=0, \quad a_{\alpha}(x) \frac{\partial V}{\partial x_{\alpha}}+U C=0
$$

and $\operatorname{det} U \neq 0$ in some domain of the point $x$ space.

Proof. Obviously,

$$
Y\left(f_{q}\right)=a_{\alpha}(x) \frac{\partial u_{q i}(x)}{\partial x_{\alpha}} E_{i}+a_{\alpha}(x) \frac{\partial v_{q}(x)}{\partial x_{\alpha}}+b_{i j} u_{q i} E_{j}+c_{i} u_{q i} .
$$

Therefore, $Y\left(f_{q}\right)=0$ if and only if

$$
a_{\alpha}(x) \frac{\partial u_{q i}}{\partial x_{\alpha}}+u_{q l} b_{l i}=0, \quad a_{\alpha}(x) \frac{\partial v_{q}}{\partial x_{\alpha}}+u_{q l} c_{l}=0
$$

for all values of $i, q=1,2, \ldots, n$. This system of equations can be rewritten in the matrix form of matrix equation (10).

The matrix $U$ is a Jacobi matrix for functions $f_{1}, f_{2}, \ldots, f_{n}$ with respect to variables $E_{1}, E_{2}, \ldots, E_{n}$. Hence, the system of these functions is functionally independent if and only if $\operatorname{det} U \neq 0$ in some domain of the point $x$ space. The theorem is proved. 
Proposition 1. Let $B \neq 0$. The matrix $U=\exp (f(x) B)$ satisfies the first equation of system (10) if and only if

$$
a_{\alpha}(x) \frac{\partial f}{\partial x_{\alpha}}=-1 .
$$

Proof. Since

$$
\frac{\partial U}{\partial x_{\alpha}}=\frac{\partial f}{\partial x_{\alpha}} U B
$$

$U$ satisfies the first equation of system (10) if and only if

$$
\left(a_{\alpha}(x) \frac{\partial f}{\partial x_{\alpha}}+1\right) U B=0 .
$$

The last equality holds if and only if

$$
a_{\alpha}(x) \frac{\partial f}{\partial x_{\alpha}}+1=0
$$

The proposition is proved.

Proposition 2. Let

$$
Y_{1}=a_{\alpha}^{(1)}(x) \frac{\partial}{\partial x_{\alpha}}+\ldots, \quad Y_{2}=a_{\alpha}^{(2)}(x) \frac{\partial}{\partial x_{\alpha}}+\ldots
$$

be operators of the form (9) and their corresponding matrices $B_{1}$ and $B_{2}$ mutually commute and are also linearly independent. The matrix $U=\exp \left(f(x) B_{1}\right) \times \exp \left(g(x) B_{2}\right)$ satisfies the system of equations

$$
a_{\alpha}^{(1)}(x) \frac{\partial U}{\partial x_{\alpha}}+U B_{1}=0, \quad a_{\alpha}^{(2)}(x) \frac{\partial U}{\partial x_{\alpha}}+U B_{2}=0
$$

if and only if

$$
\begin{aligned}
& a_{\alpha}^{(1)}(x) \frac{\partial f(x)}{\partial x_{\alpha}}+1=0, \quad a_{\alpha}^{(1)}(x) \frac{\partial g(x)}{\partial x_{\alpha}}=0, \\
& a_{\alpha}^{(2)}(x) \frac{\partial f(x)}{\partial x_{\alpha}}=0, \quad a_{\alpha}^{(2)}(x) \frac{\partial g(x)}{\partial x_{\alpha}}+1=0 .
\end{aligned}
$$

Proof. The matrix $U$ satisfies system (11) if and only if

$$
\left\{\begin{array}{l}
a_{\alpha}^{(1)}(x) \frac{\partial f(x)}{\partial x_{\alpha}} U B_{1}+a_{\alpha}^{(1)}(x) \frac{\partial g(x)}{\partial x_{\alpha}} U B_{2}+U B_{1}=0 \\
a_{\alpha}^{(2)}(x) \frac{\partial f(x)}{\partial x_{\alpha}} U B_{1}+a_{\alpha}^{(2)}(x) \frac{\partial g(x)}{\partial x_{\alpha}} U B_{2}+U B_{2}=0
\end{array}\right.
$$

Since the matrices $B_{1}$ and $B_{2}$ are linearly independent, the last system holds if and only if equations (12) hold. The proposition is proved. 
Proposition 3. Let

$$
Y_{1}=a_{\alpha}^{(1)}(x) \frac{\partial}{\partial x_{\alpha}}+\ldots, \quad Y_{2}=a_{\alpha}^{(2)}(x) \frac{\partial}{\partial x_{\alpha}}+\ldots
$$

be operators of the form (9) and their corresponding matrices $B_{1}$ and $B_{2}$ be connected by the relation $\left[B_{2}, B_{1}\right]=B_{2} B_{1}-B_{1} B_{2}=\lambda B_{1}, \lambda \neq 0$. The matrix $U=\exp \left(f(x) B_{1}\right) \exp \left(g(x) B_{2}\right)$ satisfies system of equations (11) if and only if

$$
\begin{aligned}
& a_{\alpha}^{(1)}(x) \mathrm{e}^{-\lambda g(x)} \frac{\partial f(x)}{\partial x_{\alpha}}+1=0, \quad a_{\alpha}^{(1)}(x) \frac{\partial g(x)}{\partial x_{\alpha}}=0, \\
& a_{\alpha}^{(2)}(x) \frac{\partial f(x)}{\partial x_{\alpha}}=0, \quad a_{\alpha}^{(2)}(x) \frac{\partial g(x)}{\partial x_{\alpha}}+1=0 .
\end{aligned}
$$

Proof. Since, by Campbell-Hausdorff's formula,

$$
\exp \left(\theta B_{2}\right) \cdot B_{1} \cdot \exp \left(-\theta B_{2}\right)=B_{1}+\frac{\lambda \theta}{1 !} B_{1}+\frac{(\lambda \theta)^{2}}{2 !} B_{1}+\cdots=\mathrm{e}^{\lambda \theta} B_{1},
$$

we have

$$
B_{1} \exp \left(g(x) B_{2}\right)=\mathrm{e}^{-\lambda g(x)} \exp \left(g(x) B_{2}\right) B_{1} .
$$

The matrix $U$ satisfies system (11) if and only if

$$
\left\{\begin{array}{l}
a_{\alpha}^{(1)}(x) \frac{\partial f(x)}{\partial x_{\alpha}} \mathrm{e}^{-\lambda g(x)} U B_{1}+a_{\alpha}^{(1)}(x) \frac{\partial g(x)}{\partial x_{\alpha}} U B_{2}+U B_{1}=0, \\
a_{\alpha}^{(2)}(x) \frac{\partial f(x)}{\partial x_{\alpha}} \mathrm{e}^{-\lambda g(x)} U B_{1}+a_{\alpha}^{(2)}(x) \frac{\partial g(x)}{\partial x_{\alpha}} U B_{2}+U B_{2}=0 .
\end{array}\right.
$$

By the hypothesis, $\left[B_{2}, B_{1}\right] \neq 0$, therefore, the matrices $B_{1}$ and $B_{2}$ are linearly independent. Having equated to zero the expressions at $B_{1}$ and $B_{2}$ on the left-hand sides of the equalities written down, we obtain equalities (13). The proposition is proved.

Let

$$
\vec{E}=\left(\begin{array}{c}
E_{1} \\
E_{2} \\
\vdots \\
E_{n}
\end{array}\right), \quad \vec{H}=\left(\begin{array}{c}
H_{1} \\
H_{2} \\
\vdots \\
H_{n}
\end{array}\right), \quad \vec{V}=\left(\begin{array}{c}
v_{1}(x) \\
v_{2}(x) \\
\vdots \\
v_{n}(x)
\end{array}\right) .
$$

It is easy to see that, if components of the vector-function $U \vec{E}+\vec{V}$ are linear invariants of a subalgebra $F \subset Q$ for some $n \times n$-matrix $U=U(x)$, components of the vector-function $U \vec{H}+\vec{V}$ are also linear invariants of this subalgebra $F$.

To perform symmetry reduction of system (7) to systems of ODEs we need $n$-dimensional subalgebras of the algebra $F$ that have one main invariant $\omega$ that depends only on the variables $x_{0}, x_{1}, \ldots, x_{n}$. On such subalgebras, we construct ansatzes of the form

$$
U \vec{E}+\vec{V}=\vec{M}(\omega), \quad U \vec{H}+\vec{V}=\vec{N}(\omega),
$$

where $\vec{M}(\omega), \vec{N}(\omega)$ are unknown $n$-component functions, matrices $U, \vec{V}$ are known, and $\operatorname{det} U \neq 0$ in some domain of the point $x$ space. Ansatz (14) can be presented in the form

$$
\vec{E}=U^{-1} \vec{M}(\omega)-U^{-1} \vec{V}, \quad \vec{H}=U^{-1} \vec{N}(\omega)-U^{-1} \vec{V} .
$$

We call ansatzes of the form (14) or (15) linear. In their searching for, we use the statements proved. 


\section{Solutions of system (1) that are functions of $t$ and $x_{1}$}

To obtain such solutions, one should solve system (2). As was noticed in Section 2, the invariance algebra of system $(2)$ is the affine algebra $A I G L(2, \mathbb{R})$ with the generators

$$
\begin{aligned}
& D=t \frac{\partial}{\partial t}-x_{1} \frac{\partial}{\partial x_{1}}-2 E_{1} \frac{\partial}{\partial E_{1}}-2 H_{1} \frac{\partial}{\partial H_{1}}, \quad S=x_{1} \frac{\partial}{\partial t}-E_{1}^{2} \frac{\partial}{\partial E_{1}}-H_{1}^{2} \frac{\partial}{\partial H_{1}}, \\
& T=t \frac{\partial}{\partial x_{1}}+\frac{\partial}{\partial E_{1}}+\frac{\partial}{\partial H_{1}}, \quad P_{0}=\frac{\partial}{\partial t}, \quad P_{1}=\frac{\partial}{\partial x_{1}}, \quad Z=t \frac{\partial}{\partial t}+x_{1} \frac{\partial}{\partial x_{1}} .
\end{aligned}
$$

Up to conjugacy with respect to the group of inner automorphisms the algebra $A I G L(2, \mathbb{R})$ has the following one-dimensional subalgebras:

$$
\begin{aligned}
& \langle D-\alpha Z\rangle(\alpha \geq 0, \alpha \neq 1),\left\langle D-Z-2 \alpha P_{0}\right\rangle(\alpha \in \mathbb{R}),\langle Z\rangle,\left\langle P_{1}\right\rangle, \\
& \left\langle T+\alpha P_{0}\right\rangle,\langle T+\alpha Z\rangle(\alpha \neq 0),\langle T-S+\alpha Z\rangle(\alpha \in \mathbb{R}) .
\end{aligned}
$$

Employing some of these subalgebras, let us perform reduction of system (2) to systems of ODEs, on solutions of which we shall construct the corresponding solutions of system (2) and then ones of systems (1).

3.1. $\langle D-Z\rangle: E_{1}=x_{1} M_{1}(\omega), H_{1}=x_{1} N_{1}(\omega), \omega=t$,

$$
\dot{M}_{1}+N_{1} M_{1}=0, \quad \dot{N}_{1}+M_{1} N_{1}=0 .
$$

If $M_{1}=N_{1}$, then $E_{1}=\frac{x_{1}}{t+C^{\prime}}, H_{1}=\frac{x_{1}}{t+C^{\prime}}$ and $E_{2}, E_{3}, H_{2}, H_{3}$ are arbitrary functions of $\frac{x_{1}}{t+C^{\prime}}$. If $M_{1}=N_{1}+C, C \neq 0$, then

$$
E_{1}=\frac{C x_{1}}{1-\tilde{C} \mathrm{e}^{-C t}}, \quad H_{1}=\frac{C \tilde{C} x_{1} \mathrm{e}^{-C t}}{1-\tilde{C} \mathrm{e}^{-C t}}
$$

Components $E_{2}, E_{3}$ may be arbitrary functions of $\frac{C x_{1}}{1-\tilde{C} \mathrm{e}^{-C t}}$ and components $H_{2}, H_{3}$ may be arbitrary functions of $\frac{C \tilde{C} x_{1} \mathrm{e}^{-C t}}{1-\tilde{C} \mathrm{e}^{-C t}}$.

3.2. $\left\langle T+\alpha P_{0}\right\rangle(\alpha \neq 0): E_{1}=\frac{1}{\alpha} t+M_{1}(\omega), H_{1}=\frac{1}{\alpha} t+N_{1}(\omega), \omega=x_{1}-\frac{t^{2}}{2 \alpha}$,

$$
\frac{1}{\alpha}+N_{1} \dot{M}_{1}=0, \quad \frac{1}{\alpha}+M_{1} \dot{N}_{1}=0 .
$$

The pair of functions

$$
M_{1}=\left(C \omega+C^{\prime}\right)^{\frac{1}{2}}, \quad N_{1}=-\frac{2}{\alpha C}\left(C \omega+C^{\prime}\right)^{\frac{1}{2}}
$$

is a solution of the reduced system and the corresponding solution of system (2) is of the form

$$
E_{1}=\frac{t}{\alpha}+\left[C\left(x_{1}-\frac{t^{2}}{2 \alpha}\right)+C^{\prime}\right]^{\frac{1}{2}}, \quad H_{1}=\frac{t}{\alpha}-\frac{2}{\alpha C}\left[C\left(x_{1}-\frac{t^{2}}{2 \alpha}\right)+C^{\prime}\right]^{\frac{1}{2}} .
$$


Components $E_{2}, E_{3}$ are arbitrary differentiable functions of $E_{1}$ and components $H_{2}, H_{3}$ are arbitrary differentiable functions of $H_{1}$.

$$
\begin{aligned}
& \text { 3.3. }\langle T\rangle: E_{1}=\frac{x_{1}}{t}+M_{1}(\omega), H_{1}=\frac{x_{1}}{t}+N_{1}(\omega), \omega=t, \\
& \dot{M}_{1}+\frac{1}{\omega} N_{1}=0, \quad \dot{N}_{1}+\frac{1}{\omega} M_{1}=0 .
\end{aligned}
$$

The reduced system has the general solution $M_{1}=C \omega+\frac{\tilde{C}}{\omega}, N_{1}=-C \omega+\frac{\tilde{C}}{\omega}$ and the corresponding solution of system (2) is

$$
E_{1}=\frac{x_{1}}{t}+C t+\frac{\tilde{C}}{t}, \quad H_{1}=\frac{x_{1}}{t}-C t+\frac{\tilde{C}}{t} .
$$

Components $E_{2}, E_{3}$ are arbitrary differentiable functions of $E_{1}$ and components $H_{2}, H_{3}$ are arbitrary differentiable functions of $H_{1}$.

$$
\begin{aligned}
& \text { 3.4. }\langle T-S\rangle: E_{1}=\frac{\frac{x_{1}}{t}+M_{1}(\omega)}{1-\frac{x_{1}}{t} M_{1}(\omega)}, H_{1}=\frac{\frac{x_{1}}{t}+N_{1}(\omega)}{1-\frac{x_{1}}{t} N_{1}(\omega)}, \omega=t^{2}+x_{1}^{2}, \\
& 2 \omega \dot{M}_{1}+\left(1+M_{1}^{2}\right) N_{1}=0, \quad 2 \omega \dot{N}_{1}+\left(1+N_{1}^{2}\right) M_{1}=0 .
\end{aligned}
$$

To the solution $M_{1}=N_{1}=(C \omega-1)^{-\frac{1}{2}}$ of the reduced system, there corresponds the following solution of system (2):

$$
E_{1}=H_{1}=\frac{1+\frac{x_{1}}{t}\left[C\left(t^{2}+x_{1}^{2}\right)-1\right]^{\frac{1}{2}}}{\left[C\left(t^{2}+x_{1}^{2}\right)-1\right]^{\frac{1}{2}}-\frac{x_{1}}{t}} .
$$

Components $E_{2}, E_{3}, H_{2}, H_{3}$ are arbitrary differentiable functions of $E_{1}$.

\section{Solutions of system (1) that are functions of $t, x_{1}, x_{2}$}

The problem is to constract solutions that are not equivalent to solutions depending only on $t$ and $x_{1}$. To obtain them for system (5), one should restrict oneself in reducing to employing only two-dimensional subalgebras of the algebra $A I G L(3, \mathbb{R})$ that have a zero intersection with the translation space $\left\langle P_{0}, P_{1}, P_{2}\right\rangle$. Let us give the list of such subalgebras basing on results of the classification of all subalgebras of the algebra $A I G L(3, \mathbb{R})$ with respect to affine conjugation performed in [10].

Let

$$
\begin{aligned}
& B=-\Gamma_{11}+\Gamma_{22}, \quad C=\Gamma_{21}, \quad F=-\Gamma_{12}, \quad Z=\Gamma_{11}+\Gamma_{22}, \\
& D=2 \Lambda-\Gamma_{11}-\Gamma_{22} \quad \text { (see notations (8)) }
\end{aligned}
$$

Up to affine conjugation, the algebra $A I G L(3, \mathbb{R})$ contains only the following twodimensional subalgebras that have zero intersection with the space $\left\langle P_{0}, P_{1}, P_{2}\right\rangle$ :

$$
\begin{aligned}
& \left\langle G_{1}, G_{2}\right\rangle,\left\langle G_{1}+P_{2}, G_{2}+\varepsilon P_{1}\right\rangle(\varepsilon=0,1),\left\langle G_{1}, G_{2}+P_{2}\right\rangle,\left\langle C, G_{1}\right\rangle, \\
& \left\langle C+P_{2}, G_{1}+\varepsilon P_{0}\right\rangle(\varepsilon= \pm 1),\left\langle C+P_{2}, G_{1}\right\rangle,\left\langle C+G_{2}, G_{1}\right\rangle,
\end{aligned}
$$




$$
\begin{aligned}
& \left\langle C+G_{2}, G_{1}+P_{1}\right\rangle,\langle D+Z, C\rangle,\left\langle D+Z, C+G_{2}\right\rangle,\left\langle D+Z+2 C, G_{1}\right\rangle, \\
& \left\langle D+Z+2 C+2 G_{2}, G_{1}\right\rangle,\left\langle D+Z+2 G_{1}, G_{2}\right\rangle,\left\langle D+Z+2 G_{1}, C+G_{2}\right\rangle, \\
& \left\langle D+\alpha Z, G_{1}\right\rangle,\left\langle Z, G_{1}\right\rangle,\left\langle Z-D+2 P_{1}, G_{1}\right\rangle,\left\langle Z-D+2 P_{2}, G_{1}\right\rangle, \\
& \left\langle D+3 Z, G_{1}+P_{0}\right\rangle,\left\langle Z, G_{1}+P_{2}\right\rangle,\langle D+\alpha Z, C\rangle,\langle Z, C\rangle, \\
& \left\langle Z-D+2 P_{1}, C\right\rangle,\left\langle Z-D+2 P_{1}, C+P_{2}\right\rangle,\left\langle Z-D, C+P_{2}\right\rangle, \\
& \left\langle Z+\alpha P_{0}, C+\beta P_{0}\right\rangle(\alpha, \beta=0,1 ; \alpha+\beta=1),\langle Z+C, D+Z\rangle, \\
& \left\langle D+\alpha Z+\beta C, G_{1}\right\rangle,\left\langle Z+\alpha C, G_{1}\right\rangle,\left\langle Z-D+2 C+2 P_{2}, G_{1}\right\rangle, \\
& \left\langle Z+C+P_{0}, G_{1}+P_{2}\right\rangle,\left\langle D+3 Z+2 C, G_{1}+P_{0}\right\rangle,\langle Z+\alpha C, D+Z+2 C\rangle, \\
& \left\langle B+\alpha D+(1+\alpha) Z, G_{2}\right\rangle,\left\langle B+D+2 Z, G_{2}+P_{1}\right\rangle,\left\langle B+D+2 Z, G_{2}+P_{0}\right\rangle, \\
& \left\langle B-D+P_{2}, G_{2}\right\rangle,\left\langle B+Z+P_{1}, G_{2}\right\rangle,\left\langle B+\alpha D+(1+\alpha) Z+G_{1}, G_{2}\right\rangle, \\
& \left\langle B-D+G_{1}+P_{2}, G_{2}\right\rangle,\left\langle B+Z+G_{1}, G_{2}+P_{2}\right\rangle, \\
& \left\langle B+D+2 Z+G_{1}, G_{2}+P_{1}\right\rangle,\langle B+\alpha D+\beta Z, C\rangle, \\
& \left\langle B+2 Z-D+P_{1}, C+P_{0}\right\rangle,\left\langle B+\alpha D+(1-\alpha) Z+P_{1}, C\right\rangle(\alpha \neq 0,1), \\
& \left\langle B-3 Z+P_{0}, C+P_{2}\right\rangle,\left\langle B+\alpha Z+P_{0}, C\right\rangle(\alpha \neq \pm 1), \\
& \left\langle B+\alpha D-(\alpha+3) Z, C+P_{2}\right\rangle(\alpha \neq-1,-2), \\
& \left\langle B+(\alpha-1) Z-D, C+P_{0}\right\rangle(\alpha \neq \pm 1), \\
& \left\langle B+\alpha D+(\alpha-3) Z, C+G_{2}\right\rangle,\left\langle B+2 D-Z+P_{1}, C+G_{2}\right\rangle, \\
& \left\langle B-D-4 Z, C+G_{2}+P_{0}\right\rangle,\langle F+C+\alpha Z, D+Z\rangle(\alpha \geq 0), \\
& \langle F+C+\alpha(D+Z), Z+\beta(D+Z)\rangle(\alpha \geq 0),\langle D, Z\rangle, \\
& \langle B+\alpha Z, D+Z\rangle(0 \leq \alpha<1), \\
& \langle B+\alpha(D+Z), Z+\beta(D+Z)\rangle(\alpha \geq 3 \beta+1), \\
& \langle B+D, Z\rangle,\langle 2 B+D+Z, D-Z\rangle,\left\langle 2 B+D+Z+2 P_{1}, D-Z+\alpha P_{1}\right\rangle, \\
& \left\langle 2 B+D+Z, Z-D+2 P_{1}\right\rangle,\left\langle F+C+P_{0}, Z+\alpha P_{0}\right\rangle(\alpha \geq 0),\left\langle F+C, Z+P_{0}\right\rangle \text {. }
\end{aligned}
$$

From this list, we exclude the subalgebra $\left\langle C, G_{1}\right\rangle$, the rank of which is equal to 1 with respect to the variables $t, x_{1}, x_{2}$.

Let us present some examples of symmetry reduction of system (5), performed with the help of given subalgebras.

4.1. $\left\langle G_{1}, G_{2}+P_{2}\right\rangle: E_{1}=\frac{x_{1}}{t}+M_{1}(\omega), E_{2}=\frac{x_{2}}{t+1}+M_{2}(\omega)$,

$$
\begin{aligned}
& H_{1}=\frac{x_{1}}{t}+N_{1}(\omega), H_{2}=\frac{x_{2}}{t+1}+N_{2}(\omega), \omega=t, \\
& \begin{cases}N_{1}+\omega \dot{M}_{1}=0, & N_{2}+(\omega+1) \dot{M}_{2}=0, \\
M_{1}+\omega \dot{N}_{1}=0, & M_{2}+(\omega+1) \dot{N}_{2}=0 .\end{cases}
\end{aligned}
$$


The general solution of the reduced system

$$
\begin{array}{ll}
M_{1}=C_{1} \omega+\frac{C_{2}}{\omega}, & M_{2}=C_{3}(\omega+1)+\frac{C_{4}}{\omega+1}, \\
N_{1}=-C_{1} \omega+\frac{C_{2}}{\omega}, & N_{2}=-C_{3}(\omega+1)+\frac{C_{4}}{\omega+1},
\end{array}
$$

is associated with the following solution of system (5):

$$
\begin{array}{ll}
E_{1}=\frac{x_{1}}{t}+C_{1} t+\frac{C_{2}}{t}, & E_{2}=\frac{x_{2}}{t+1}+C_{3}(t+1)+\frac{C_{4}}{t+1}, \\
H_{1}=\frac{x_{1}}{t}-C_{1} t+\frac{C_{2}}{t}, & H_{2}=\frac{x_{2}}{t+1}-C_{3}(t+1)+\frac{C_{4}}{t+1} .
\end{array}
$$

In this case, $E_{3}$ is an arbitrary differentiable function of $E_{1}$ and $E_{2}$ and $H_{3}$ is an arbitrary differentiable function of $H_{1}$ and $H_{2}$.

$$
\begin{aligned}
& \text { 4.2. }\left\langle C+P_{2}, G_{1}+\varepsilon P_{0}\right\rangle(\varepsilon= \pm 1): E_{1}=\varepsilon t+M_{1}(\omega)+x_{2} M_{2}(\omega), E_{2}=M_{2}(\omega) \text {, } \\
& H_{1}=\varepsilon t+N_{1}(\omega)+x_{2} N_{2}(\omega), \quad H_{2}=N_{2}(\omega), \omega=t^{2}-2 \varepsilon x_{1}+\varepsilon x_{2}^{2}, \\
& \begin{cases}\varepsilon-2 \varepsilon N_{1} \dot{M}_{1}+N_{2} M_{2}=0, & N_{1} \dot{M}_{2}=0, \\
\varepsilon-2 \varepsilon M_{1} \dot{N}_{1}+M_{2} N_{2}=0, & M_{1} \dot{N}_{2}=0 .\end{cases}
\end{aligned}
$$

The solution of the reduced system $M_{1}=N_{1}=0, M_{2}=M(\omega), N_{2}=-\frac{\varepsilon}{M(\omega)}$, where $M(\omega)$ is an arbitrary nonzero function, is associated with the solution of system (1):

$$
\begin{aligned}
& E_{1}=\varepsilon t+x_{2} M(\omega), \quad H_{1}=\varepsilon t-\varepsilon x_{2} M^{-1}(\omega), \\
& E_{2}=M(\omega), \quad H_{2}=-\varepsilon M^{-1}(\omega), \quad \omega=t^{2}-2 \varepsilon x_{1}+\varepsilon x_{2}^{2}, \\
& E_{3}=g\left(\varepsilon t+x_{2} M(\omega), M(\omega)\right), \quad H_{3}=h\left(\varepsilon t-\varepsilon x_{2} M^{-1}(\omega),-\varepsilon M^{-1}(\omega)\right),
\end{aligned}
$$

where $g, h$ are arbitrary differentiable functions.

The solution $M_{1}=0, M_{2}=C_{1}, N_{1}=C_{2}, N_{2}=-\frac{\varepsilon}{C_{1}}$, is associated with the solution of system (5)

$$
E_{1}=\varepsilon t+C_{1} x_{2}, \quad E_{2}=C_{1}, \quad H_{1}=\varepsilon t-\frac{\varepsilon}{C_{1}} x_{2}+C_{2}, \quad H_{2}=-\frac{\varepsilon}{C_{1}} .
$$

For it,

$$
\begin{aligned}
& E_{3}=g\left(x_{2}+\frac{\varepsilon}{C_{1}} t, x_{1}-\frac{\varepsilon}{2} t^{2}+\frac{\varepsilon}{C_{1}} t x_{2}+\frac{t^{2}}{2 C_{1}^{2}}-C_{2} t\right), \\
& H_{3}=h\left(x_{2}-C_{1} t, x_{1}-C_{1} t x_{2}+\frac{C_{1}^{2}-\varepsilon}{2} t^{2}\right),
\end{aligned}
$$

where $g, h$ are arbitrary differentiable functions.

4.3. $\left\langle C+P_{2}, G_{1}\right\rangle: E_{1}=\frac{x_{1}}{t}-\frac{x_{2}^{2}}{2 t}+M_{1}(\omega)+x_{2} M_{2}(\omega), E_{2}=M_{2}(\omega)$, 


$$
\begin{aligned}
& H_{1}=\frac{x_{1}}{t}-\frac{x_{2}^{2}}{2 t}+N_{1}(\omega)+x_{2} N_{2}(\omega), H_{2}=N_{2}(\omega), \omega=t, \\
& \begin{cases}\omega \dot{M}_{1}+N_{1}+\dot{M}_{2} \dot{N}_{2} \omega=0, & \dot{M}_{2}=0, \\
\omega \dot{N}_{1}+M_{1}+\dot{M}_{2} \dot{N}_{2} \omega=0, & \dot{N}_{2}=0 .\end{cases}
\end{aligned}
$$

The reduced system has the general solution $M_{1}=C_{3} \omega+\frac{C_{4}}{\omega}, N_{1}=-\left(C_{1} C_{2}+C_{3}\right) \omega+\frac{C_{4}}{\omega}$, $M_{2}=C_{1}, N_{2}=C_{2}$. It is associated with the following solution of system (5):

$$
\begin{aligned}
& E_{1}=\frac{x_{1}}{t}-\frac{x_{2}^{2}}{2 t}+C_{1} x_{2}+C_{3} t+\frac{C_{4}}{t}, \quad E_{2}=C_{1}, \\
& H_{1}=\frac{x_{1}}{t}-\frac{x_{2}^{2}}{2 t}+C_{2} x_{2}-\left(C_{1} C_{2}+C_{3}\right) t+\frac{C_{4}}{t}, \quad H_{2}=C_{2} .
\end{aligned}
$$

Components $E_{3}, H_{3}$ are given by the formulas:

$$
\begin{aligned}
& E_{3}=g\left(x_{2}-C_{2} t, x_{1}-\left(\frac{C_{2}^{2}}{2}-C_{1} C_{2}-C_{3}\right) t^{2}+C_{4}-\frac{\left(x_{2}-C_{2} t\right)^{2}}{2}\right), \\
& H_{3}=h\left(x_{2}-C_{1} t, x_{1}-\left(\frac{C_{1}^{2}}{2}+C_{3}\right) t^{2}+C_{4}-\frac{\left(x_{2}-C_{1} t\right)^{2}}{2}\right),
\end{aligned}
$$

where $g, h$ are arbitrary differentiable functions.

\section{References}

[1] Fushchych W.I., New nonlinear equations for electromagnetic field having the velocity different from c, Dopov. Ukr. Acad. Sci., 1992, N 4, 24-27.

[2] Rosen G., Conformal transformation matrix for fields, Ann. Phys. (USA), 1973, V.77, N 2, 452-453.

[3] Kapitanskii L.V., Group analisys of the Navier-Stokes and Euler equations in the presence of the rotational symmetry and new exact solutions for these equations, Dokl. Acad. Sci. of USSR, 1978, V.243, N 4, 901-904 (in Russian).

[4] Fushchych W., Shtelen W. and Serov N., Symmetry Analysis and Exact Solutions of Equations of Nonlinear Mathematical Physics, Dordrecht, Kluwer Academic Publishers, 1993.

[5] Fairlie D.B. and Leznov A.N., General solution of the universal equation in $n$-dimensional space, J. Nonlin. Math. Phys., 1994, V.1, N 4, 333-339.

[6] Fushchych W.I. and Boyko V.M., Lowering of order and general solutions for some classes of equations of mathematical physics, Dopov. Nation. Acad. Sci. of Ukraine, 1996, N 9, 43-48 (in Ukrainian).

[7] Fushchych W., Tsyfra I. and Boyko V., Nonlinear representations for Poincaré and Galilei algebras and nonlinear equations for electromagnetic fields, J. Nonlin. Math. Phys., 1994, V.1, N 2, 210-221.

[8] Fushchych W.I., Symmetry in problems of mathematical physics, In: Algebraic-theoretic researches in mathemetical physics, Kiev, Inst. of Mathematics of Acad. Sci. Ukrain. SSR, 1981, 6-28 (in Russian).

[9] Fushchych W.I., On symmetry and exact solutions of multidimensional nonlinear wave equations, Ukr. Math. J., 1987, V.39, N 1, 116-123 (in Russian).

[10] Barannyk A.F., Moskalenko Yu.D. and Fushchych W.I., Subalgebras of the affine algebra $A I G L(3, R)$, Kiev, 1989, 32p. (Preprint, Acad. Sci. of USSR, Inst. of Mathematics; 89.65). 